$$
\text { May 1-5, } 1995 \text { Dallas, Texas }
$$

\title{
CRYSTALLINE BEAM PROPERTIES AS PREDICTED FOR THE STORAGE RINGS ASTRID and TSR*
}

\author{
Jie Wei, Brookhaven National Laboratory, P.O. Box 5000, Upton, New York 11973-5000, USA \\ Xiao-Ping Li, BIOSYM Technologies Inc., 9685 Scranton Rd. San Diego, CA92121, USA \\ Andrew Sessler, Lawrence Berkeley Laboratory, Berkeley, California 94720, USA
}

\begin{abstract}
Employing a previously developed formalism ${ }^{1}$, we have performed ground-state and melting calculations of the expected crystalline beams in ion storage rings ASTRID and TSR.
\end{abstract}

\section{INTRODUCTION}

During recent years, significant progress has been made in understanding the formation of crystalline beams in circular accelerators and storage rings. ${ }^{1-4}$ No crystalline beam exists in a constant gradient storage ring, but in an alternating gradient ( $\mathrm{AG}$ ) ring crystalline beams exist at all densities as long as the beam energy is smaller than the transition energy of the machine. The ground-state structure depends upon the machine lattice property, the beam energy, and the beam intensity (line density). At low density, the ground state is a one-dimensional (1D) chain with particles equally spaced along the azimuthal axis. As the density increases, the ground state first becomes a $2 \mathrm{D}$ zigzag in the plane of weaker transverse focusing, and then becomes 3D single- and multi-shell helices. The maximum spatial density of the crystal, on the other hand, is determined only by the machine lattice property and beam energy, independent of the beam intensity.

When circulating in an AG-focusing machine, the total energy of the beam is not a constant of motion. As a result, the crystalline beam will gradually heat up and eventually melt if not refrigerated. Previously, we have shown that if the machine lattice periodicity is lower than twice the maximum betatron tune, heat will generally transfer into the system extremely fast so that a crystalline beam can not last a meanirgful period of time (except at very low density). On the other hand, if the lattice periodicity is significantly higher than twice the maximum betatron tune, the heat transfer is slow, and the crystalline beam can last for a long time. ${ }^{1,4}$

\section{CRYSTALLINE BEAM IN THE ASTRID}

ASTRID is a charged-ion storage ring of circumference $C_{0}=40 \mathrm{~m}$. Laser cooling has been used to reduce the velocity spread of the beam, currently achieving a rest frame temperature $T=1 \mathrm{mK}$ in longitudinal dimension. The lattice has a super-periodicity of 4 .

Employing the previously developed formalism, ${ }^{1}$ we perform the molecular dynamics (MD) calculation and analysis with the $100 \mathrm{keV}^{7} \mathrm{Li}^{+}$ion beam. The transverse tunes

\footnotetext{
- Work performed under the auspice of the U.S. Department of Energy, Office of High Energy and Nuclear Physics under Contract No. DE-AC03-76SF00098.
}

of the machine are $\nu_{x}=2.62$ and $\nu_{y}=1.13$. The characteristic distance is ${ }^{1}$

$$
\xi \equiv\left(\frac{q^{2} r_{0} \rho^{2}}{A \beta^{2} \gamma^{2}}\right)^{1 / 3} \approx 2.2 \times 10^{-5} \mathrm{~m}
$$

where $r_{0}=e^{2} / m_{0} c^{2}=1.53 \times 10^{-18} \mathrm{~m}$ is the proton classical radius, $\rho=1.2 \mathrm{~m}$ is the bending radius, $\beta c=5.54 \times 10^{-3} c$ is the beam velocity, $\gamma=\left(1-\beta^{2}\right)^{-1 / 2}, q=1$ is the charge, and $A \approx 7$ is the atomic mass number.

At low density, the ground state is a $1 D$ chain. MD calculation shows that the transition from $1 \mathrm{D}$ to $2 \mathrm{D}$ occurs when the inter-particle distance $\Delta_{z} \approx 5.5$ in reduced units, ${ }^{1}$ i.e. when the number of particles in the machine is

$$
N=\frac{\gamma \bar{C}_{0}}{\Delta_{z} \xi} \approx 3.4 \times 10^{5}
$$

When the total number of particles exceeds this value, the ground state first becomes a $2 \mathrm{D}$ zig-zag in the vertical plane where the focusing is relatively weak. Fig. 1 shows the tra-

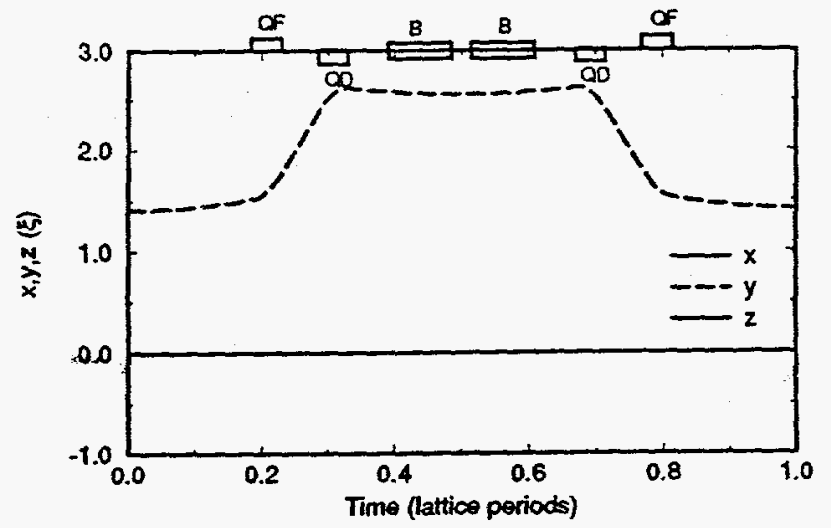

Figure. 1. A vertical zig-zag structure at density $\Delta_{z}=$ 3 formed with the ASTRID lattice. The displacement in both $x$ and $z$ is zero at all time. B, QF, and QD denote dipole, focusing quadrupole, and defocusing quadrupole, respectively.

jectory of the particle with positive $y$ in such a structure at $\Delta_{z}=3$ (i.e. $N=6.1 \times 10^{5}$ ). The breathing of the structure can be seen as the particles encounter the focusing and defocusing elements of the machine.

Due to the AG focusing, the crystalline structure absorbs energy (heat) from the lattice. The dots in Fig. 2 shows the heating rate as a function of the temperature $T$ in the reduced units ${ }^{1}$ for a $1 \mathrm{D}$ crystal of density $\Delta_{z}=10(N=$ $\left.1.8 \times 10^{5}\right)$. The conventional rest-frame temperature can be conveniently obtained by the relation

$$
T_{\text {rest }}=\frac{A m_{0} c^{2} \beta^{2} \gamma^{2} \xi^{2}}{2 k_{B} \rho^{2}} T=0.38 T(K),
$$




\section{DISCLAIMER}

This report was prepared as an account of work sponsored by an agency of the United States Government. Neither the United States Government nor any agency thereof, nor any of their employees, makes any warranty, express or implied, or assumes any legal liability or responsibility for the accuracy, completeness, or usefulness of any information, apparatus, product, or process disclosed, or represents that its use would not infringe privately owned rights. Reference herein to any specific commercial product, process, or service by trade name, trademark, manufacturer, or otherwise does not necessarily constitute or imply its endorsement, recommendation, or favoring by the United States Government or any agency thereof. the views and opinions of authors expressed herein do not necessarily state or reflect those of the United States Government or any agency thereof. 


\section{DISCLAIMER}

Portions of this document may be illegible in electronic image products. Images are produced from the best available original document. 


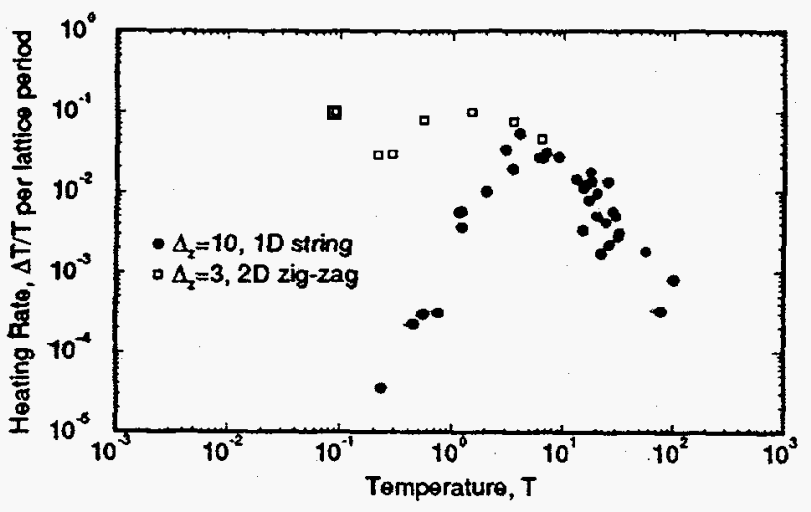

Figure. 2. Heating rates as functions of the temperature $T$ for a $1 D\left(\Delta_{z}=10\right)$ and a $2 D\left(\Delta_{z}=3\right)$ ground state in the ASTRID lattice.

where $k_{B}$ is the Boltzmann constant. At low temperature, the heating rate is low. Fig. 3 shows that although the

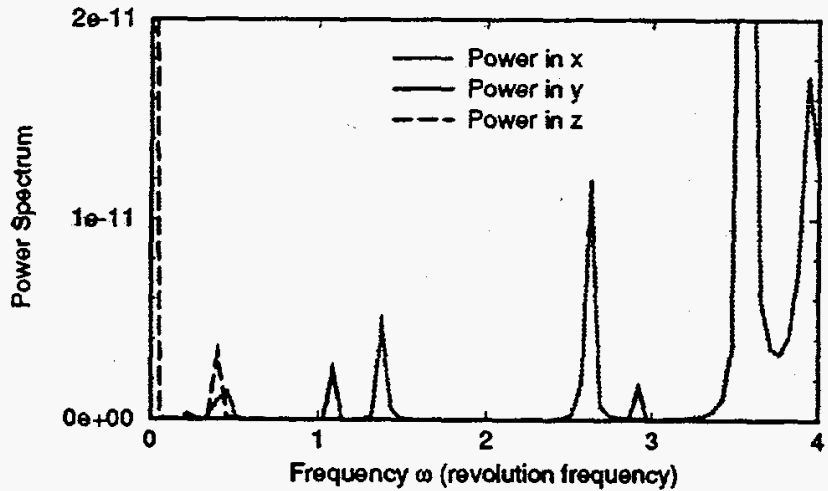

Figure. 3. Power spectrum for the $1 \mathrm{D}\left(\Delta_{z}=10\right)$ structure in the ASTRID lattice at extremely low temperature. The machine tunes are $\nu_{x}=2.62, \nu_{x}=1.13$.

horizontal tune $\left(\nu_{x}=2.62\right)$ is larger than half the lattice periodicity (4), the phonon spectrum does not extend to twice the revolution frequency $\left(\omega_{0}\right)$, thus avoiding the twophonon process which leads to immediate heat-up. ${ }^{4}$

As the temperature increases, the heating rate increases due to the increased probability of multi-phonon processes. The maximum heating rate is $\Delta T / T \approx 4 \times 10^{-2}$ per lattice period, which corresponds to an e-folding growth time of $0.15 \mathrm{~ms}$. The break up of the crystal occurs at the temperature that corresponds to the maximum heating rate, i.e. $T \approx 4$ (total rest-frame temperature $T_{\text {rest }}=1.5 \mathrm{~K}$ ). As the temperature increases further, the heating rate decreases due to the reducing spatial density. The MD calculation quantitatively agrees with the conventional intrabeam scattering calculation at the high-temperature limit (approximately $T>20$ )

The squares in Fig. 2 shows that the 2D structure heats up quickly even at very low temperature. Fig. 4 shows that the phonon spectrum of the 2D structure extends over a broad range including $2 \omega_{0}$. The immediate heat up occurs at any temperature through the two-phonon process.

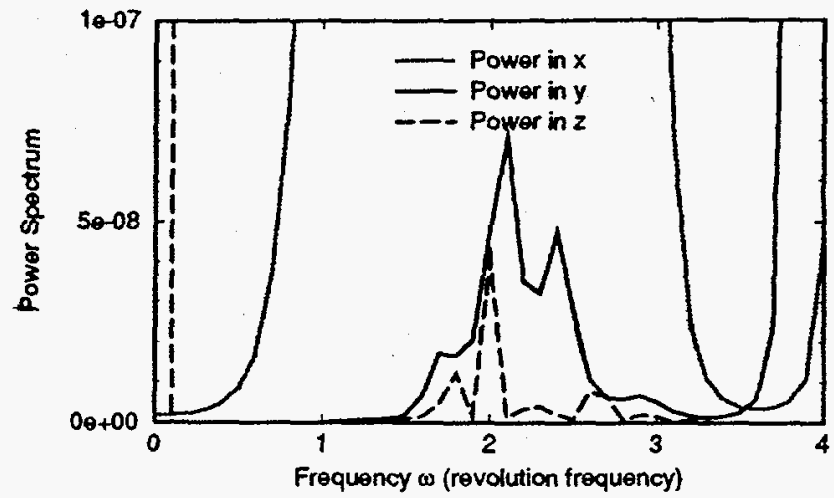

Figure. 4. Power spectrum for the 2D $\left(\Delta_{z}=3\right)$ structure in the ASTRID lattice at extremely low temperature.

\section{CRYSTALLINE BEAM IN THE TSR}

The TSR is an ion storage ring of circumference $C_{0}=$ $55.4 \mathrm{~m}$. The lattice has a super-periodicity of 2 . We perform MD calculation with the $7.3 \mathrm{MeV}{ }^{9} \mathrm{Be}^{+}$ion beam. The transverse tunes of the machine are $\nu_{x}=2.57$ and $\nu_{y}=2.21$. The characteristic distance is $\xi \approx 5.1 \times 10^{-6} \mathrm{~m}$ $(\rho=1.15 \mathrm{~m})$.

At low density, the ground state is again a $1 D$ chain. The transition from $1 \mathrm{D}$ to $2 \mathrm{D}$ occurs approximately at $\Delta_{z}=6$, or $N=1.8 \times 10^{6}$. When the total number of particles $N$ exceeds this value, the ground state becomes a zig-zag. The structure makes one rotation per lattice period around its longitudinal axis, having a horizontal orientation in the middle of the straight sections with particle separation of $2.5 \xi$, and a vertical orientation in the middle of the bending region with particle separation $8.7 \xi$. The rotation is produced by the shear motion when the particle crosses the bending magnetic field and is shown in Fig. 5 for $\Delta_{z}=5$ $\left(N=2.2 \times 10^{6}\right)$. The direction of the rotation is determined

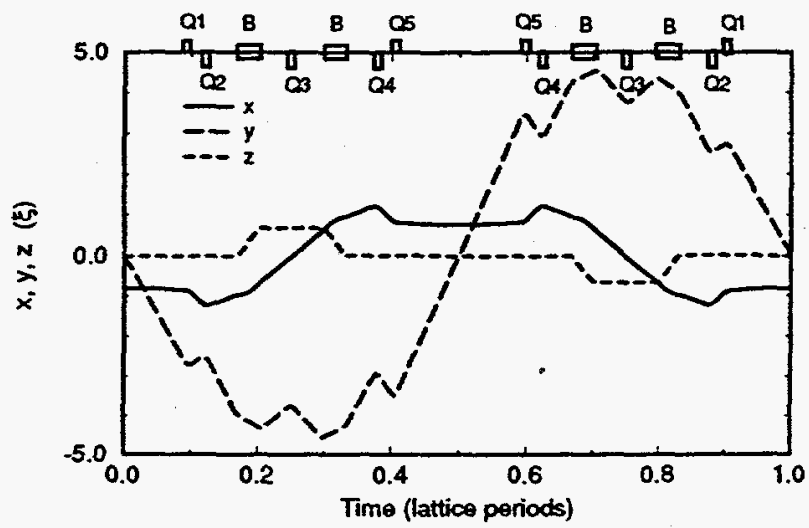

Figure. 5. A rotating zig-zag structure at density $\Delta_{z}=5$ formed with the TSR lattice with the displacements in $x$, $y$, and $z$ changing with time during one lattice period.

by the orientation of the bending magnetic field.

The dots and squares in Fig. 6 show the heating rates as functions of the temperature $T$ in reduced units ${ }^{1}$ for a 1D crystal of density $\Delta_{z}=10\left(N=1.1 \times 10^{6}\right)$ and the $2 \mathrm{D}$ rotating zig-zag of $\Delta_{z}=5$. The conventional 


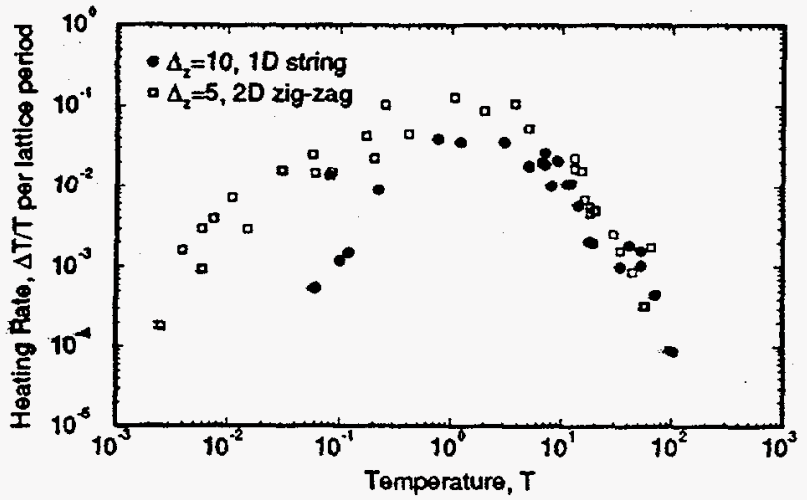

Figure. 6. Heating rates as functions of the temperature $T$ for a 1D $\left(\Delta_{z}=10\right)$ and a $2 \mathrm{D}\left(\Delta_{z}=5\right)$ ground-state structure in the TSR lattice.

rest-frame temperature can be obtained by the relation $T_{\text {rest }}=1.64 T(\mathrm{~K})$. At low temperature, the heating rate for both structure is low. Fig. 7 shows the phonon spec-

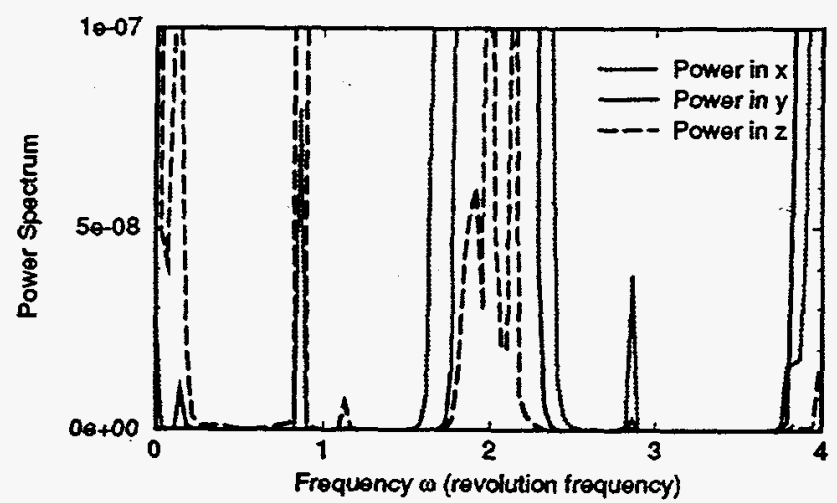

Figure. 7. Power spectrum for the $2 \mathrm{D}\left(\Delta_{z}=5\right)$ structure in the TSR lattice at extremely low temperature. The machine tunes are $\nu_{x}=2.57, \nu_{x}=2.21$.

trum of the 2D structure. The gigantic peak at $2 \omega_{0}$ results from the periodic ground-state motion, while the spread is partially due to the finite length of steps used for spectrum analysis. Although the transverse tunes are larger than half the lattice periodicity (2), the phonon spectrum luckily avoids the dangerous points (integral multiple of $\omega_{0}$ ) which would lead to immediate heat-up.

The maximum heating rate $\Delta T / T \approx 1.2 \times 10^{-1}$ per lattice period (e-folding growth time of $0.018 \mathrm{~ms}$ ) for the 2D state is about twice of the $1 D$ state $\Delta T / T \approx 6 \times 10^{-2}$. The break up of the crystal occurs at the temperature that corresponds to the maximum heating rate, i.e. $T \approx 1.3$ $\left(T_{\text {rest }}=2.1 \mathrm{~K}\right)$.

\section{QUANTUM LIMIT}

The classical MD calculation fails only at very low temperature when quantum effects become important. The threshold temperature $T_{s h}$ is given by the relation

$$
k_{B} T_{s h} \sim \hbar \omega_{\max }
$$

where $\omega_{\max }$ is the maximum phonon frequency of the crystal. For the ASTRID 1D $\left(\Delta_{z}=10\right)$ structure, $T_{s h} \approx$ $5 \times 10^{-6} \mathrm{~K}$, as shown in Fig. 8 .

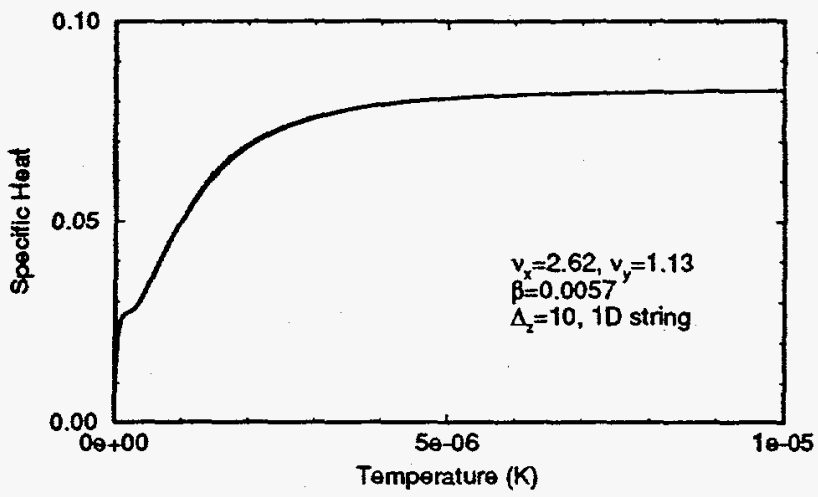

Figure. 8. Specific heat (in reduced units) for the 1D $\left(\Delta_{z}=10\right)$ structure in the ASTRID lattice.

\section{CONCLUSIONS AND DISCUSSION}

Employing the previously developed formalism ${ }^{1}$, we have performed MD calculations of the expected crystalline beams in ASTRID and TSR. The crystalline structure and the melting conditions depend on the beam energy, density, and the machine properties. For both ASTRID and TSR, we find that $1 D$ and $2 D$ crystals can be formed when the total rest-frame temperature is less than about $1 \mathrm{~K}$.

For $1 \mathrm{D}$ and occasional $2 \mathrm{D}$ crystalline structure, the maximum rate at which the crystalline state absorbs energy from the lattice occurs at the (break-up) temperature when the crystal starts to form (or break). The maximum rate is found to be linearly proportional to the beam intensity. With a given cooling rate, crystalline state of sufficiently low density can in principle be achieved if the external system noise is sufficiently. low.

Because of the relatively low machine lattice periodicity, crystals of $2 \mathrm{D}$ or higher dimension typically absorbs energy from the lattice and heat up extremely fast. In order to obtain crystalline beam of complex structure, storage rings should be designed with lattice periodicity significantly higher than twice the maximum transverse tune.

Acknowledgment We thank J. Hangst and D. Habs for many useful discussions, and A. Draeseke for making the computer program more efficient.

\section{REFERENCES}

1. J. Wei, X-P Li, and A.M. Sessler, Phys. Rev. Lett. 73, 3089 (1994).

2. J.P. Schiffer and P. Kienle, Z. Phys. A321, 181 (1985); J.P. Schiffer and O. Poulsen, Europhys. Lett. 1, 55 (1986); J.P. Schiffer, Phys.Rev. Lett. 57, 1133 (1986).

3. For example, J. Beebe-Wang, et al. Nucl. Instrum. Methods B79, 806 (1993); CRYSTAL LNL-INFN (REP) 59/92, Laboratori Nazional di Legnaro.

4. X-P Li, A.M. Sessler, and J. Wei, Proc. European Part. Accel. Conf. 1994, London, p.1379 (1994). 